\title{
Treatment Landscape for Patients with HER2-Positive Metastatic Breast Cancer: A Review on Emerging Treatment Options
}

This article was published in the following Dove Press journal: Cancer Management and Research

\author{
Simon Peter Gampenrieder ${ }^{1,2}$ \\ Vanessa Castagnaviz' \\ Gabriel Rinnerthaler ${ }^{1,2}$ \\ Richard Greil ${ }^{1,2}$ \\ 'Department of Internal Medicine III with \\ Haematology, Medical Oncology, \\ Haemostaseology, Infectiology and \\ Rheumatology, Oncologic Center, \\ Salzburg Cancer Research Institute - \\ Laboratory for Immunological and \\ Molecular Cancer Research (SCRI- \\ LIMCR), Paracelsus Medical University \\ Salzburg, Salzburg, Austria; ${ }^{2}$ Cancer \\ Cluster Salzburg, Salzburg, Austria
}

Correspondence: Richard Greil Illrd Medical Department, Paracelsus Medical University Salzburg, Müllner Hauptstraße 48, Salzburg 5020, Austria Tel +4357255-2580 I

Fax +4357255-25999

Email r.greil@salk.at

\begin{abstract}
The prognosis of HER2-positive metastatic breast cancer (MBC) has radically changed in recent years and continues to improve due to the broad application of effective therapies like monoclonal antibodies and small molecules targeting HER2. Persistent dependency of tumor cells on the oncogene HER2, on one hand, as well as low expression levels in healthy tissue, on the other hand, make this protein an ideal target for anti-cancer therapy. New HER2 targeting strategies including targeted delivery of cytotoxic drugs via HER2 receptor have been developed. Recently, the US Food and Drug Administration (FDA) approved three new drugs for the treatment of HER2-positive MBC: the antibody-drug conjugate trastuzumab deruxtecan and the two tyrosine kinase inhibitors neratinib and tucatinib. Here, we summarize recent publications and developments of novel anti-HER2 therapies like monoclonal antibodies with improved properties compared to trastuzumab and bispecific antibodies, which bind two different HER-epitopes or bring T cells closer to tumor cells. Furthermore, novel antibody-drug conjugates and small molecules against HER2 are discussed. These developments coupled with new combination strategies (eg, with CDK4/6 inhibitors or immunotherapy) will change the treatment landscape for patients with HER2positive MBC very soon and will hopefully further improve clinical outcomes.
\end{abstract}

Keywords: margetuximab, bispecific antibodies, trastuzumab duocarmazine, trastuzumab deruxtecan, neratinib, tucatinib, immunotherapy, CDK4/6 inhibitors

\section{Introduction}

Based on gene expression assays, breast cancer can be divided into at least four socalled intrinsic subgroups with distinct biological, clinical and prognostic behavior. ${ }^{1}$ In clinical practice, immunohistochemical markers are used as surrogates in order to guide therapeutic decisions. ${ }^{2}$ The most frequent subtype - about $70 \%$ of all breast cancers - is hormone receptor (HR)-positive and human epidermal growth factor receptor 2 (HER2)-negative, followed by the HER2-positive subtypes (HR-positive or negative, together about $15 \%$ ) and the so-called triple-negative subtype, lacking the expression of estrogen receptor (ER), progesterone receptor (PR) and HER2 (about 15\%). ${ }^{3}$

The mainstay of treatment of MBC is endocrine therapy, chemotherapy, targeted therapies and immunotherapy. Unfortunately, efficacy of endocrine therapy as well as immunotherapy is not (yet) satisfying in HER2-positive disease and chemotherapy is associated with substantial toxicity. Therefore, in the past, HER2-positive MBC was associated with short responses and limited survival. ${ }^{4}$ Fortunately, since 
the introduction of trastuzumab, the first humanized monoclonal antibody (mAb) targeting HER2, the prognosis of HER2-positive MBC has considerably improved over the last two decades. In a Phase 3 trial, the median overall survival (OS) significantly improved from 20.3 to 25.1 months (relative risk [RR] 0.80; 95\% CI 0.64-1.00; $\mathrm{P}=0.046$ ) when trastuzumab was combined with first-line chemotherapy compared to chemotherapy alone. ${ }^{5}$ Median OS in palliative first-line was even higher (between 36 and 41 months) when trastuzumab was combined with docetaxel, one of the most potent chemotherapeutic drugs for MBC. ${ }^{6-8}$ This success stimulated the development of further anti-HER2 targeting drugs like the orally applied tyrosine kinase inhibitor lapatinib, which exerts intracellular activity against both EGFR (epidermal growth factor receptor) and HER2. Lapatinib monotherapy or in combination with capecitabine achieved a breakthrough in the treatment of HER2-positive patients with brain metastases that had relapsed after whole-brain irradiation, since it crosses the blood-brain barrier. ${ }^{9}$ The next milestone in the treatment of HER2-positive MBC was the addition of pertuzumab to trastuzumab and chemotherapy. Pertuzumab is a humanized mAb with another mechanism of action when compared with trastuzumab. It targets a different epitope of the HER2 extracellular domain and thereby prevents hetero-dimerization of HER2 with other HER receptors, particularly with HER3. Both trastuzumab and pertuzumab stimulate antibody-dependent, cellmediated cytotoxicity (ADCC). ${ }^{10}$ In the CLEOPATRA phase 3 trial, the addition of pertuzumab to the combination of docetaxel and trastuzumab as first-line treatment improved median OS by almost 16 months (56.5 vs 40.8 months; hazard ratio $[\mathrm{HR}] \quad 0.68 ; 95 \%$ CI $0.56-0.84$; $\mathrm{P}<0.001)$.

Besides the abovementioned progress in palliative firstline, new treatment options in later lines have been approved in recent years based on survival improvements in several Phase III trials. T-DM1 represents the first antibody-drug conjugate (ADC) approved for the treatment of MBC. ADCs are biopharmaceutical drugs consisting of a monoclonal antibody covalently bound to a cytotoxic drug (called payload) via a synthetic linker. ${ }^{11}$ ADCs combine the advantages of specific targeting through the antibody and high cytotoxicity by the attached chemotherapeutic drug. The phase 3 registration trials EMILIA $^{12}$ and THERESA, ${ }^{13}$ comparing T-DM1 with capecitabine plus lapatinib and with treatment according to physician's choice, respectively, established T-DM1 as today's standard of care for second-line treatment of HER2-positive MBC. In case of early progression after or during adjuvant therapy, T-DM1 is the therapy of choice for palliative first-line treatment.

These targeted treatment options for HER2-positive MBC have radically attenuated the hopelessness of this aggressive disease. Nevertheless, HER2-positive MBC is still an incurable disease and further research is necessitated to help women progressing on treatment, especially in case of brain metastases.

In the last year, several data on new therapeutic options for HER2-positive MBC have been published. This review summarizes the developments of novel anti-HER2 therapies such as modified monoclonal antibodies, tyrosine kinase inhibitors, antibody-drug conjugates as well as bispecific antibodies targeting two different epitopes. Furthermore, new combination strategies with CDK4/6 inhibitors and immune-checkpoint inhibitors are discussed (summarized in Table 1 and Figure 1), thereby illustrating the emerging treatment landscape for patients with HER2positive MBC.

\section{Novel Anti-HER2 Antibodies Margetuximab (MGAH22, MacroGenics)}

Margetuximab is a chimeric anti-HER2 mAb with an Fc domain genetically modified for improved binding to Fcgamma receptors (Fc $\gamma \mathrm{Rs}$ ), aiming at increasing ADCC. Compared to trastuzumab, margetuximab shows higher affinity to both alleles of the stimulatory FcyR IIIA (coded by $C D 16 A$ ) and lower affinity to the inhibitory Fc $\gamma$ R IIB (coded by $C D 32 B){ }^{14}$

The SOPHIA phase III trial compared margetuximab (15 mg/kg every 3 weeks IV) plus chemotherapy $(\mathrm{n}=266)$ with trastuzumab plus chemotherapy $(n=270)$ in pretreated patients with HER2-positive MBC. Chemotherapy was chosen by the investigator out of four options: capecitabine, eribulin, gemcitabine or vinorelbine. Patients had received at least two prior anti-HER2 therapies including pertuzumab and one to three treatment lines in the metastatic setting. Brain metastases were allowed if they were treated and stable. In the second interim OS analysis, presented at the 2019 San Antonio Breast Cancer Symposium (SABCS), the median OS in the intention-to-treat (ITT) population was 21.6 months with margetuximab compared with 19.8 months with trastuzumab (HR 0.89 ; 95\% CI 0.69-1.13; $\mathrm{P}=0.33) .{ }^{15}$ Despite a missing OS advantage, margetuximab plus chemotherapy significantly improved progression-free 
Table I Overview of New Treatment Options for HER2-Positive MBC, Their Phase of Development and Currently Running Trials. Phase 2 and 3 Trials with Published Results are Highlighted in Bold

\begin{tabular}{|c|c|c|c|}
\hline & Substance Group & $\begin{array}{l}\text { Phase of Development } \\
\text { (ClinicalTrials.gov Identifier) }\end{array}$ & Study Arms and Purpose \\
\hline Margetuximab & $\begin{array}{l}\text { Monoclonal } \\
\text { antibody }(\mathrm{mAb})\end{array}$ & $\begin{array}{l}\text { Phase } 3 \\
\text { SOPHIA } \\
\text { SCT024927II }\end{array}$ & $\begin{array}{l}\text { Margetuximab + chemotherapy vs Trastuzumab }(T)+ \\
\text { chemotherapy }\end{array}$ \\
\hline ZW25 & Bispecific antibody & $\begin{array}{l}\text { Phase I NCT02892 I } 23 \\
\text { Phase I b/2 NCT04276493 } \\
\text { Phase } 2 \text { NCT04224272 }\end{array}$ & $\begin{array}{l}\text { Part I: dose escalation Part 2/3: ZW } 25 \text { alone and in combination } \\
\text { with chemotherapy } \\
\text { Cohort I: ZW } 25+\text { docetaxel } \\
\text { Part I: safety and tolerability Part 2: efficacy of ZW } 25+ \\
\text { palbociclib + fulvestrant }\end{array}$ \\
\hline $\begin{array}{l}\text { Zenocutuzumab } \\
\text { (MCLA-I28) }\end{array}$ & Bispecific antibody & Phase $2^{15}$ NCT0332198I & Cohort I: Zenocutuzumab $+\mathrm{T} \pm$ vinorelbine \\
\hline $\begin{array}{l}\text { GBRI302 (ISB } \\
\text { I302) }\end{array}$ & $\begin{array}{l}\text { T-cell dependent } \\
\text { (CD3) bispecific } \\
\text { antibody }\end{array}$ & Phase I/2 NCT03983395 & Dose escalation \\
\hline BTRC40I7A & $\begin{array}{l}\text { T-cell dependent } \\
(C D 3) \text { bispecific } \\
\text { antibody }\end{array}$ & Phase I NCT03448042 & Dose escalation \\
\hline PRS-343 & $\begin{array}{l}\text { T-cell dependent } \\
\text { (CDI37) bispecific } \\
\text { antibody }\end{array}$ & $\begin{array}{l}\text { Phase I NCT0333056 I } \\
\text { Phase I NCT03650348 }\end{array}$ & $\begin{array}{l}\text { Dose escalation } \\
\text { Dose escalation in combination with atezolizumab }\end{array}$ \\
\hline $\begin{array}{l}\text { Trastuzumab } \\
\text { deruxtecan (DS- } \\
8201 \text { a) }\end{array}$ & $\begin{array}{l}\text { Antibody-drug } \\
\text { conjugate }\end{array}$ & $\begin{array}{l}\text { Phase I NCT02564900 } \\
\text { Phase } 2 \text { DESTINY-Breast0 I } \\
\text { 16 NCT03248492 } \\
\text { Phase } 3 \text { DESTINY-Breast02 } \\
\text { NCT03523585 } \\
\text { Phase } 3 \text { DESTINY-Breast03 } \\
\text { NCT03529IIO } \\
\text { Phase } 3 \text { DESTINY Breast04 } \\
\text { NCT03734029 }\end{array}$ & $\begin{array}{l}\text { Dose escalation } \\
\text { Part I: recommended part 2-dose Part 2: safety and efficacy } \\
\text { as single agent in T-DMI resistant/refractory patients } \\
\text { T-deruxtecan vs T + Capecitabine }(C) \text { vs Lapatinib }(L)+C \\
\text { T-deruxtecan vs T-DMI } \\
\text { T-deruxtecan vs physician's choice treatment }\end{array}$ \\
\hline $\begin{array}{l}\text { Trastuzumab } \\
\text { duocarmazine } \\
\text { (SYD985) }\end{array}$ & $\begin{array}{l}\text { Antibody-drug } \\
\text { conjugate }\end{array}$ & $\begin{array}{l}\text { Phase I NCT022777I7 } \\
\text { Phase } 3 \text { TULIP NCT03262935 }\end{array}$ & $\begin{array}{l}\text { Dose escalation } \\
\text { T duocarmazine vs physician's choice treatment }\end{array}$ \\
\hline BAT800I & $\begin{array}{l}\text { Antibody-drug } \\
\text { conjugate }\end{array}$ & $\begin{array}{l}\text { Phase I NCT04I892II } \\
\text { Phase } 3 \text { NCT04I85649 }\end{array}$ & $\begin{array}{l}\text { Dose escalation and safety } \\
\text { BAT800I vs } L+C\end{array}$ \\
\hline $\begin{array}{l}\text { RC48-ADC } \\
\text { (hertuzumab-vc- } \\
\text { MMAE) }\end{array}$ & $\begin{array}{l}\text { Antibody-drug } \\
\text { conjugate }\end{array}$ & $\begin{array}{l}\text { Phase I NCT0288I I } 38 \\
\text { Phase Ib NCT03052634 } \\
\text { Phase } 2 \text { NCT03500380 }\end{array}$ & $\begin{array}{l}\text { Dose escalation and safety } \\
\text { Recommended phase } 2 \text { dose } \\
\text { RC48-ADC vs L + C }\end{array}$ \\
\hline ARX788 & $\begin{array}{l}\text { Antibody-drug } \\
\text { conjugate }\end{array}$ & Phase I CTR2017III62 & Dose escalation, safety and efficacy \\
\hline ALT-P7 & $\begin{array}{l}\text { Antibody-drug } \\
\text { conjugate }\end{array}$ & Phase I NCT0328I824 & Dose escalation, safety and efficacy \\
\hline Neratinib & $\begin{array}{l}\text { Irreversible pan- } \\
\text { HER tyrosine kinase } \\
\text { inhibitor }\end{array}$ & $\begin{array}{l}\text { Phase } 2^{17} \text { NCT0030078I } \\
\text { Phase } 2^{18} \text { NCT00777I II }\end{array}$ & $\begin{array}{l}\text { Efficacy of neratinib in T-pretreated and T-non pretreated } \\
\text { patients } \\
\text { Neratinib vs } L+C\end{array}$ \\
\hline
\end{tabular}

(Continued) 
Table I (Continued).

\begin{tabular}{|c|c|c|c|}
\hline & Substance Group & $\begin{array}{l}\text { Phase of Development } \\
\text { (ClinicalTrials.gov Identifier) }\end{array}$ & Study Arms and Purpose \\
\hline & & $\begin{array}{l}\text { Phase } 2 \text { NEfERT- } \\
T^{19} \text { NCT009I50I8 } \\
\text { Phase I b/2 NSABP FB- } \\
10^{20} \text { NCT02236000 } \\
\text { Phase } 2 \text { TBCRC } \\
022^{21} \text { NCT0I } 494662 \\
\text { Phase } 3 \\
\text { NALA }{ }^{22} \text { NCT0 } 1808573\end{array}$ & $\begin{array}{l}\text { Neratinib + paclitaxel vs T + Paclitaxel } \\
\text { Dose escalation and efficacy of Neratinib + T-DMI } \\
\text { Cohort 3a/3b: efficacy of neratinib + C in CNS-MBC } \\
\text { Neratinib + C vs L + C }\end{array}$ \\
\hline Pyrotinib & $\begin{array}{l}\text { Irreversible pan-HER } \\
\text { tyrosine kinase } \\
\text { inhibitor }\end{array}$ & $\begin{array}{l}\text { Phase I NCTOI } 937689 \\
\text { Phase } 2^{23} \text { NCT02422 I } 99 \\
\text { Phase } 3 \\
\text { PHOEBE }\end{array}$ & $\begin{array}{l}\text { Dose escalation, safety and efficacy } \\
\text { Pyrotinib + C vs } \mathbf{L}+\mathbf{C} \\
\text { Pyrotinib + C vs } \mathbf{L}+\mathbf{C}\end{array}$ \\
\hline Tucatinib & $\begin{array}{l}\text { Selective HER2 } \\
\text { tyrosine kinase } \\
\text { inhibitor }\end{array}$ & $\begin{array}{l}\text { Phase Ib NCT02025I92 } \\
\text { Phase } 2 \\
\text { HER2CLIMB }{ }^{25} \text { NCT026I4794 }\end{array}$ & $\begin{array}{l}\text { Efficacy and Safety of tucatinib }+\mathbf{T} \pm \text { capecitabine } \\
\text { Tucatinib }+\mathbf{T}+\mathbf{C} \text { vs placebo }+\mathbf{T}+\mathbf{C}\end{array}$ \\
\hline Abemaciclib & CDK4/6 inhibitor & $\begin{array}{l}\text { Phase } 2 \\
\text { MonarcHER }{ }^{26} \text { NCT0267523I }\end{array}$ & $\begin{array}{l}\text { Abemaciclib }+\mathbf{T}+\text { fulvestrant vs Abemaciclib }+\mathbf{T} \text { vs } \mathbf{T}+ \\
\text { physician's choice treatment }\end{array}$ \\
\hline Palbociclib & CDK4/6 inhibitor & $\begin{array}{l}\text { Phase } 2 \\
\text { PATRICIA }{ }^{27} \text { NCT02448420 }\end{array}$ & $\begin{array}{l}\text { Part I: Palbociclib + T } \pm \text { letrozole Part 2: Palbociclib + T + } \\
\text { endocrine therapy vs } \mathrm{T}+\text { physician's choice treatment } \\
\text { (T-DMI or chemotherapy) }\end{array}$ \\
\hline Pembrolizumab & $\begin{array}{l}\text { Checkpoint } \\
\text { inhibitor (CPI), anti- } \\
\text { PD-I mAb }\end{array}$ & $\begin{array}{l}\text { Phase Ib-2 } \\
\text { PANACEA }{ }^{28} \text { NCT02I } 29556\end{array}$ & $\begin{array}{l}\text { Safety and efficacy of Pembrolizumab }+T \text { in T-resistant } \\
\text { MBC }\end{array}$ \\
\hline Atezolizumab & $\begin{array}{l}\text { CPI, anti-PD-LI } \\
\text { mAb }\end{array}$ & $\begin{array}{l}\text { Phase } 2 \\
\text { KATE2 }^{29,30} \text { NCT02924883 } \\
\text { Phase } 3 \text { NCT03199885 }\end{array}$ & $\begin{array}{l}\text { T-DMI + atezolizumab vs T-DMI + placebo } \\
\text { T + pertuzumab }(P)+\text { paclitaxel + atezolizumab vs } T+P+\text { paclitaxel } \\
\text { +placebo }\end{array}$ \\
\hline Avelumab & $\begin{array}{l}\text { CPI, anti-PD-LI } \\
m A b\end{array}$ & Phase 2 AVIATOR NCT034I4658 & $\mathrm{T}+$ vinorelbine + avelumab \pm utomilumab ( 4 IBB/CDI 37 agonist) \\
\hline
\end{tabular}

survival (PFS), as assessed by the study investigators (median PFS 5.7 vs 4.4 months; HR 0.71 ; 95\% CI 0.58-0.86; $\mathrm{P}<0.001$ ), overall response rate (ORR $25.2 \%$ vs $13.7 \%$; $\mathrm{P}<0.001)$ as well as clinical benefit rate $(\mathrm{CBR}=\mathrm{CR}+\mathrm{PR}$ $+\mathrm{SD}$ for $\geq 6$ months: $48.1 \%$ vs $35.6 \% ; \mathrm{P}<0.001)$ compared to the control arm. The advantage, however, seemed to be limited to patients with a CD16A-185F genotype (FF or FV; $86 \%$ of the population), while in patients homozygous for the VV allele, the trastuzumab-based regimen performed better. This exploratory analysis, however, is hypothesis generating only because the results may have been influenced by imbalances of patient characteristics. ${ }^{15}$ Based on these data, a biologics license application (BLA) was submitted to the FDA by the developing company in December 2019.

\section{Bispecific Antibodies}

Bispecific mAbs currently under development for the treatment of HER2-positive solid tumors target two different epitopes either on HER2 (or on HER2 and HER3) or HER2, on the one hand, and an epitope on T cells, on the other hand. These $\mathrm{T}$ cell-dependent bispecific (TDB) antibodies are designed to redirect $T$ cells to attack HER2expressing cancer cells. 


\section{Emerging treatment options for metastatic HER2 positive breast cancer under investigation}
1 Novel anti-HER2 antibodies
2 Bispecific antibodies
3 T-cell dependend bispecific antibodies
Antibody-drug conjugates (ADC)

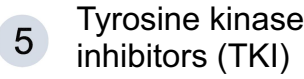
6 CDK 4/6 inhibitors
7 Immune checkpoint inhibitors
4

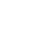

$(2$

2

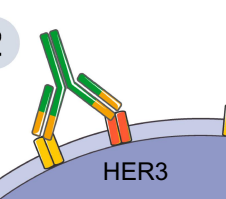

1

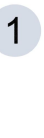

T-cell

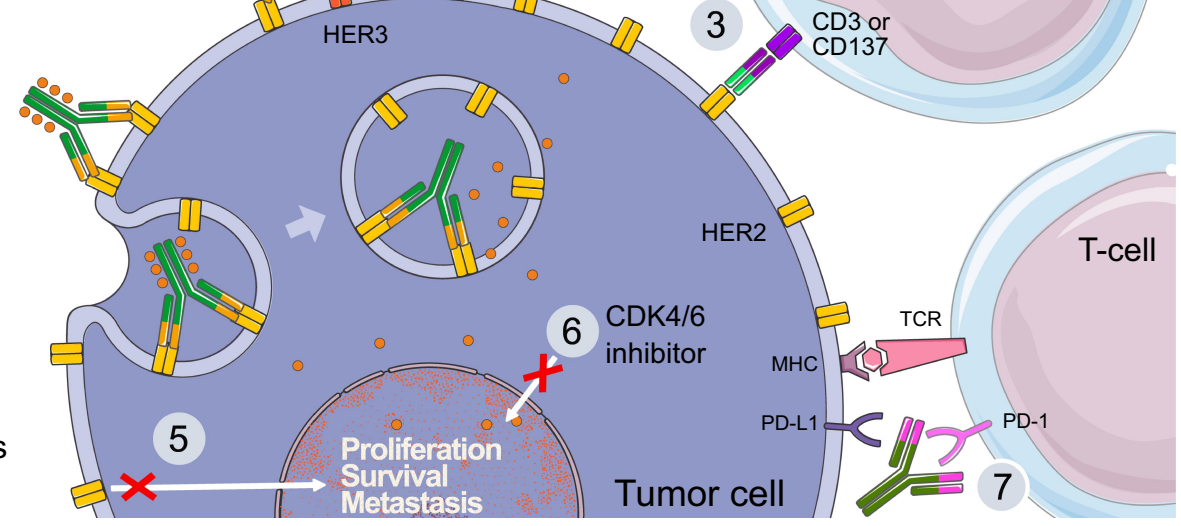

Figure I New treatment strategies for HER2-positive MBC discussed in this review. (I) Novel anti-HER2 antibodies bind to the extracellular domain of HER2 and activate the antibody-dependent cell-mediated cytotoxicity (ADCC) more efficiently than trastuzumab; (2) Bispecific antibodies bind to two different epitopes either on HER2 or on HER2 and HER3; (3) T-cell dependent bispecific (TDB) antibodies bind to HER2 on one hand and to an epitope on T cells on the other hand thereby activating the adaptive immune system; (4) Antibody-drug conjugates (ADCs) use the specificity of HER2-directed antibodies to deliver a highly potent chemotherapy directly into the tumor cell; (5) Tyrosine kinase inhibitors (TKIs) are orally available small molecules that block the intracellular domain of HER2 and other receptors of the HER family; (6) CDK4/6 inhibitors block the common final path of several growth factor signaling pathways, including the one of HER2; (7) Currently available immune checkpoint inhibitors target the programmed cell death protein I (PD-I) or its ligand PD-LI and release the "break" in the adaptive immune system.

\section{ZW25 (Zymeworks Inc.)}

ZW25 targets simultaneously two different HER2 domains: ECD4, the binding site of trastuzumab and ECD2, the binding site of pertuzumab. The latter mechanism increases tumor-cell binding by the antibody, HER2 receptor clustering and internalization as well as blockade of ligand-dependent and independent tumor cell growth. Of note, preclinical studies demonstrated anti-tumor activity also in tumor models with low HER2 expression. ${ }^{16}$

Results of a Phase I dose-escalation study were presented at the 2018 Annual Meeting of the American Society of Clinical Oncology (ASCO), which included patients with HER2 expressing tumors that had progressed during standard therapies, including trastuzumab, pertuzumab and T-DM1 in breast cancer and trastuzumab in gastroesophageal cancer. ${ }^{17}$ In the heavily pretreated breast cancer cohort $(n=20)$ with a median of five prior HER2-targeting therapies, ORR and disease control rate (DCR) were $33 \%$ and $50 \%$, respectively. ZW25 was generally well tolerated with only one patient experiencing grade 3 AEs. The most common grade 1 and 2 AEs were diarrhea and infusion reactions. Dose-limiting toxicities or treatment-related serious adverse events (SAEs) were not reported, neither treatment discontinuations due to AEs. ${ }^{17}$

The following trials are currently ongoing: a Phase 1/2 trial investigating the combination of ZW25 and docetaxel as first-line treatment of HER2-positive MBC (ClinicalTrials.gov identifier: NCT04276493) and a Phase 2 trial combining ZW25 with the CDK4/6 inhibitor palbociclib and fulvestrant for patients with HER2-positive, hormone-receptor[HR]-positive MBC (NCT04224272).

\section{Zenocutuzumab (MCLA- I28; Merus)}

Zenocutuzumab is a bispecific humanized full-length IgG1 antibody that binds both HER2 and HER3. It has a dual mechanism of action: 1) it inhibits the HER2:HER3 signaling axis and 2) it shows enhanced ADCC activity. Zenocutuzumab was developed to overcome resistance to HER2- or EGFR-targeted therapies.

The first-in-human phase 1/2 study (NCT02912949) included 8 patients with heavily pretreated HER2-positive MBC and a median of 5.5 prior treatment lines in the metastatic setting. Zenocutuzumab showed an encouraging CBR of $70 \%$ in this population. One patient achieved a partial remission (PR; 12.5\%). ${ }^{18}$ The main toxicities 
were infusion-related reactions (IRR), mostly of grade 1 and 2 , diarrhea, rash, and fatigue (each $13 \%$ of patients).

In a Phase II trial (NCT03321981) 39 patients who had progressed on trastuzumab/pertuzumab and T-DM1 were treated with zenocutuzumab plus trastuzumab and vinorelbine. ${ }^{19}$ The CBR at 24 weeks, the primary endpoint (evaluable in 37 patients), was 35.1\% (95\% CI 22.2-50.0). The ORR was $18.9 \%$ including one patient with a CR. The main toxicities of this regimen were neutropenia (54\% G3/ 4) and diarrhea (all grades 61\%, G3/4 4\%). IRR were reported in $18 \%$ of patients. ${ }^{19}$ In a second cohort patients with HR-positive/HER2-low MBC refractory to a CDK4/6 inhibitor plus endocrine therapy were treated with zenocutuzumab in combination with the same endocrine therapy. In this cohort, a CBR of $16.7 \%$ was achieved including one patient with a PR. ${ }^{20}$

Besides the abovementioned activity findings in HER2dependent tumors, zenocutuzumab has shown to be active in tumors driven by neuregulin-1 (NRG-1) fusion proteins by preventing their binding to HER $3 .^{21}$

\section{T Cell-Dependent Bispecific (TDB) Antibodies}

Several TDBs are currently under development including antibodies targeting HER2 and the T-cell co-receptor CD3 (GBR1302 = ISB1302; Glenmark Pharmaceuticals [NCT 03983395] and BTRC4017A; Genentech [NCT0344 8042]), HER2 and the key costimulatory immunoreceptor CD137 (PRS-343; Pieris Pharmaceuticals, Inc. [NCT03 330561 and NCT03650348]) as well as the truncated form of HER2 p95HER2 and CD3 (p95HER2-TCB ${ }^{22}$ ). However, up to date, no efficacy results from clinical trials are available for the latter drugs. Hence, efficacy data are eagerly awaited before integrating TDB antibodies into the treatment landscape of HER2-positive MBC.

\section{Antibody-Drug Conjugates (ADC) Trastuzumab Deruxtecan (DS-820la; Daiichi Sankyo)}

Trastuzumab deruxtecan is an ADC composed of trastuzumab, a cleavable tetrapeptide-based linker and deruxtecan, a potent topoisomerase I inhibitor as payload. Compared with T-DM1, trastuzumab deruxtecan has a higher drug-to-antibody ratio (approximately 8 vs 3-4) and the payload shows a higher drug membrane permeability, which potentially allows for a bystander killer effect on neighboring cancer cells. The short half-life of deruxtecan minimizes systemic exposure. The linker is stable in plasma and is cleaved selectively by cathepsins that are upregulated in tumor cells. ${ }^{23}$

In the two-part, open-label, single-group, multicenter, phase 2 study DESTINY-Breast01, trastuzumab deruxtecan was evaluated in patients with HER2-positive MBC who had undergone previous treatment with T-DM1. ${ }^{24}$ Patients with treated and stable brain metastases were allowed to be included. Most patients were heavily pretreated with a median number of previous cancer treatment lines of 6 (range 2-27); 52.7\% of patients had HR-positive tumors. In part 1 of the study, three different doses of trastuzumab deruxtecan were investigated: $5.4 \mathrm{mg} / \mathrm{kg}$, $6.4 \mathrm{mg} / \mathrm{kg}$ and $7.4 \mathrm{mg} / \mathrm{kg}$. The lowest dose was chosen for part 2 of the trial. In total, 184 patients were enrolled receiving trastuzumab deruxtecan at a dose of $5.4 \mathrm{mg} / \mathrm{kg}$ every three weeks. The safety and efficacy data of this cohort were presented at the 2019 SABCS and were simultaneously published in the New England Journal of Medicine. ${ }^{24}$ All patients had undergone pretreatment with T-DM1 and trastuzumab, 66\% had received pertuzumab and 54\% additional anti-HER targeting treatments. In the ITT analysis, the ORR was $60.9 \%$, including $6 \%$ complete remissions $(\mathrm{CR})$, the $\mathrm{CBR}(=\mathrm{CR}+\mathrm{PR}+\mathrm{SD}$ for $\geq 6$ months) was $76.1 \%$ and the DCR $(=\mathrm{CR}+\mathrm{PR}+\mathrm{SD})$ 97.3\%. The median PFS was 16.4 months (95\% CI 12.7 vs not reached), the median duration of response was 14.8 months (95\% CI 13.8-16.9) and the median OS was not reached at the time point of data cutoff.

The most frequent toxicities observed in the DESTINY-Breast01 trial were nausea (all grades 78\%, G3 8\%), fatigue (all grades 50\%, G3 6\%) and alopecia (all grades 48\%, G3 0.5\%). Neutropenia grade 3 was recorded in $20 \%$ of patients, the potentially dangerous grade 4 neutropenia, however, was infrequent $(1.1 \%)$. Only one patient $(0.5 \%)$ developed a grade 4 thrombocytopenia. Of note, in $14 \%$ of patients treated with trastuzumab deruxtecan, an interstitial lung disease (ILD) was diagnosed. The latter events were nearly all of grade 1 or 2 (11\%); however, one patient had grade 3 ILD and 4 patients $(2.2 \%)$ died due to severe ILD according to the independent adjudication.

In summary, this first phase 2 trial showed an impressive efficacy signal of trastuzumab deruxtecan in HER2positive $\mathrm{MBC}$, despite the extensive pretreatment status of most patients. The relatively high incidence of potentially life-threatening ILD requires careful monitoring and has to be further investigated in the running phase 3 trials 
DESTINY-Breast02, -03 and -04 (NCT03523585, NCT03529110 and NCT03734029, respectively). Based on the data of the phase 1 trial $^{25}$ and the DESTINYBreast01 trial, the FDA approved trastuzumab deruxtecan in December 2019 for the treatment of HER2-positive MBC pretreated with two or more anti-HER2-based regimens in the metastatic setting.

\section{Trastuzumab Duocarmazine (SYD985; Synthon Biopharmaceuticals BV)}

Trastuzumab duocarmazine is a third-generation ADC based on trastuzumab, a cleavable linker as well as the payload duocarmycin, which is present as an inactive prodrug (seco-duocarmycin-hydroxybenzamide-azaindole = seco-DUBA). The binding to HER2 triggers endocytosis. The linker is cleaved in- and outside the tumor cell by proteases and releases the membrane-permeable active molecule duocarmycin allowing a significant bystander killer effect. Duocarmycin binds to the minor groove of DNA, causing irreversible DNA alkylation and cell death not only in dividing cells but also in nondividing cells in the tumor microenvironment.

To date, the results of a phase 1 dose-escalation and dose-expansion trial are published showing activity of trastuzumab duocarmazine in patients with heavily pretreated solid tumors and an immunohistochemically measured expression level of HER2 classified as $1+{ }^{26}$ In total, 146 patients were enrolled in the dose-expansion phase and treated with the recommended phase 2 dose defined in the dose-escalation part of the trial $(1.2 \mathrm{mg} / \mathrm{kg}$ IV every 3 weeks). The most common treatment-related AEs (grades $1-4)$ were fatigue (33\%), conjunctivitis $(31 \%)$ and dry eyes (31\%). Almost two thirds of patients $(71 \%)$ had at least one ocular adverse event, with grade 3 events reported in $7 \%$ of patients. Four patients in the whole cohort developed pneumonitis, one of them even a grade 4 pneumonitis (1\%). Gastrointestinal and hematologic AEs were infrequent and of low grade. The ORR in the HER2-positive breast cancer cohort assessable for response $(\mathrm{n}=48)$ was 33\% (all PR) and median PFS was 7.6 months (95\% CI 4.2-10.9). Interestingly, patients with low HER2-expression (IHC 1+ or $2+$ without HER2 gene amplification; $n=47$ ) also achieved objective responses $(28 \%$ in $\mathrm{HR}+, 40 \%$ in $\mathrm{HR}-$ $\mathrm{MBC}) .{ }^{26}$ Based on these results, the FDA granted fast track designation for trastuzumab duocarmazine in January 2018.

Currently, trastuzumab duocarmazine is investigated in the phase 3 TULIP trial recruiting patients with HER2- positive MBC pretreated with at least two HER2-targeting treatment regimens for locally advanced or metastatic disease or progression during or after T-DM1 treatment (NCT03262935). Eligible patients are randomly assigned (2:1) to receive trastuzumab duocarmazine or treatment according to physician's choice.

\section{BAT800 I (Bio-Thera Solutions)}

Analogous to T-DM1, BAT8001 is an ADC linking trastuzumab to a maytansine derivative. BAT8001, however, uses a novel uncleavable linker with the goal to reduce systemic side effects. Apart from that, BAT8001 shows similar pharmacodynamics as T-DM1. ${ }^{27}$

Data from a phase 1 trial were presented at the 2019 American Association for Cancer Research (AACR) Annual Meeting. ${ }^{28}$ In total, 29 patients were recruited, 27 patients received treatment. The most common and severe AEs were thrombocytopenia and AST elevation, similar to the toxicity profile of T-DM1. The preliminary efficacy assessment found one CR (3.7\%), five PR (18.5\%) and five disease stabilizations $(18.5 \%) .^{28}$

Based on these data, a randomized phase 3 trial was initiated for patients with HER2-positive MBC who previously had received trastuzumab monotherapy or in combination with taxanes (NCT04185649). Patients are randomized between BAT8001 and lapatinib plus capecitabine. The design of this trial, fully conducted in China, will make the interpretation of its results difficult, as the control arm represents a former therapy standard and will allow only an indirect comparison with T-DM1.

\section{RC48 (RC48-ACD, Hertuzumab-vc- MMAE; RemeGen)}

RC48 is a novel ADC, conjugating the spindle toxin monomethyl auristatin $\mathrm{E}$ (MMAE) to the humanized antiHER $2 \mathrm{mAb}$ hertuzumab via a cleavable linker. This linker was designed to only release the toxin once inside a lysosome. Hertuzumab binds specifically and with high affinity to HER2, but not to other members of the human epidermal growth factor receptor family (EGFR, HER3 or HER4). ${ }^{29}$

Recently, a pooled analysis of two phase 1 studies conducted in China was presented showing an ORR of $31 \%(22 / 70)$, a CBR of $39 \%$ and a median PFS of 5.8 months in heavily pretreated HER2-positive MBC patients. ${ }^{30}$ The most common treatment-related AEs were elevated liver enzymes (71\%), hematologic toxicities 
(67\%), neurotoxicity (57\%) and gastrointestinal disorders (44\%), which were mostly classified as grade 1 or 2 . Grade 3 or 4 AEs were reported in $41 \%$ of patients, representing mainly neutropenia (21\%), asthenia (16\%) and leukopenia $(10 \%) .{ }^{30} \mathrm{RC} 48$ has been further investigated in urothelial cancer. In a single-arm phase 2 trial, 43 patients with metastatic urothelial cancer, immunohistochemically $2+$ or $3+$ for HER2, received RC48 at the recommended phase 2 dose. The ORR and DCR were 61\% (95\% CI $44.4 \%-75.0 \%)$ and $91 \%(39 / 43)$, respectively. ${ }^{31}$

A phase 2 study in HER2-positive MBC, which evaluates the efficacy of RC48 versus capecitabine plus lapatinib in patients who had received a taxane and trastuzumab is underway (NCT03500380). Similar to the phase 3 trial with BAT8001 (NCT04185649), the design of this trial comes with considerable limitations, since BAT8001 is not compared with the actual standard therapeutic T-DM1.

\section{ARX788 (Ambrx, Inc.)}

ARX788 consists of a HER2-targeting mAb, which includes a non-natural amino acid at the pre-determined site on the heavy chain. This non-natural amino acid is specifically and non-cleavable conjugated to the cytotoxic payload AS269, a highly potent tubulin inhibitor. The mean drug-antibody ratio is 1.9 . In a breast cancer xenograft model resistant to trastuzumab (JIMT-1), ARX788 was significantly more effective in inducing tumor shrinkage than T-DM1 at equivalent doses. ${ }^{32}$

In phase 1, 45 Chinese women with HER2-positive MBC received different doses of ARX788. No doselimiting toxicities or treatment-related SAEs were observed. The main side effects were ocular and pulmonary toxicities. Ocular events were mostly mild to moderate and reversible. Three participants experienced grade 2 drug-related pneumonitis, each of them resolved after steroid treatment and ARX788 treatment was re-initiated in a reduced dose. Amongst 42 evaluated participants, the ORR was $31 \%(13 / 42)$. In patients receiving the recommended phase 2 dose, ORR was $42 \%(5 / 12)$.

\section{ALT-P7 (Alteogen)}

ALT-P7 is a novel ADC in which two molecules of MMAE are conjugated to a trastuzumab variant through a cleavable linker. In a Korean first-in-human phase 1 trial, 27 patients with heavily pretreated HER2-positive MBC were treated with different doses of ALT-P7. ${ }^{33}$ The most common treatment-related grade $3 / 4$ adverse event was neutropenia in $14.8 \%$ of patients. Other adverse events were myalgia, pruritus and fatigue, mostly of grade 1 . The recommended phase 2 dose of ALT-P7 was determined to be $4.5 \mathrm{mg} / \mathrm{kg}$. In 25 patients evaluable for response, the disease control rate $(\mathrm{DCR}=\mathrm{CR}+\mathrm{PR}+\mathrm{SD})$ was $72 \%$; the $\mathrm{CBR}(=\mathrm{CR}+\mathrm{PR}+\geq 6$ months $\mathrm{SD}$ ) was $32 \%$ including two patients with PR (8\%). The median PFS at doses ranging from 2.4 to $4.8 \mathrm{mg} / \mathrm{kg}$ was 6.2 months (95\% CI 2.5-9.9 months). ${ }^{33}$ Based on these encouraging results, ALT-P7 will continue to phase 2. Furthermore, its applicability in other HER2-positive carcinomas such as gastric, urothelial or biliary tract cancer will be evaluated.

The development of several ADCs for HER2-positive breast cancer had to be stopped either due to toxicity issues or due to the competitive environment for HER2-targeted therapies; eg, ADCT-502 (ADC Therapeutics), XMT-1522 (Mersana Therapeutics, Inc.) and MEDI4276 (Medimmune, AstraZeneca). Further, ADCs are currently investigated in phase 1 trials including patients with HER2-positive MBC (A166, DHES0815A, B003, LCB14-0110, SHR-A1201, DP303c, ZW49 and MT-5111).

\section{Tyrosine Kinase Inhibitors (TKI) Neratinib (Puma Biotechnology)}

Neratinib is an orally available irreversible pan-HER TKI, targeting HER1, HER2 and HER4. Based on the results of the adjuvant ExteNET trial, neratinib is approved by the FDA and by the European Medicines Agency (EMA) for the extended adjuvant treatment of patients with early HER2-positive breast cancer, after one year of adjuvant trastuzumab. ${ }^{34}$

Notwithstanding, neratinib has also demonstrated relevant clinical activity in the metastatic setting. In a twocohort phase II trial, neratinib as single agent achieved an ORR of $56 \%$ in the trastuzumab-naïve and $24 \%$ in the trastuzumab-pretreated cohort, respectively. ${ }^{35}$ Conversely, in a direct comparison of neratinib with capecitabine plus lapatinib, non-inferiority could not be proven. ${ }^{36}$ Further studies succeeded in demonstrating the efficacy of neratinib in combination with other drugs, such as paclitaxel (NEfERT-T trial), ${ }^{37}$ T-DM1 (NSABP FB-10 trial) ${ }^{38}$ and capecitabine (phase II TBCRC 02 and phase III NALA trial)..$^{39,40}$

The NALA phase 3 trial compared neratinib plus capecitabine with lapatinib plus capecitabine in patients with HER2-positive MBC, who have received at least two prior anti-HER2-based regimens. ${ }^{40}$ The risk of disease 
progression or death was reduced by $24 \%$ in the neratinibarm compared to the lapatinib-arm (HR 0.76; 95\% CI $0.63-0.93 ; \mathrm{P}=0.006$ ) with a prolongation of PFS by 2.2 months in the restricted means analysis ( 8.8 vs 6.6 months; $\mathrm{P}=0.0003$ ). The greatest benefit was observed in patients with non-visceral metastases (HR 0.44) and HR-negative tumors (HR 0.42). Although not statistically significant, a favorable trend towards an improved OS (HR 0.88; 95\% CI $0.72-1.07 ; \mathrm{P}=0.2086)$ and $\mathrm{ORR}(32.8 \%$ vs $26.7 \%$; $\mathrm{P}=0.1201$ ) was observed. The duration of response was significantly longer in the neratinib-arm (8.5 vs 5.6 months, HR $0.50 ; 95 \%$ CI $0.33-0.74 ; \quad \mathrm{P}=0.0004)$. Furthermore, the time to intervention for central nervous system disease was delayed in the neratinib-arm (overall cumulative incidence $22.8 \%$ vs $29.2 \% ; \quad \mathrm{P}=0.043$ ). Treatment-associated AEs were comparable between the two arms, except for a higher rate of grade 3 diarrhea in the neratinib arm (24.4\% vs $12.5 \%)$ although treatment discontinuation rate due to diarrhea was similar between the two arms (about 2.5\%). ${ }^{40}$

Based on these results, the FDA approved neratinib in combination with capecitabine for patients with advanced HER2-positive breast cancer who have received at least 2 prior anti-HER2-based regimens in the metastatic setting.

\section{Pyrotinib (Jiangsu Hengrui Pharmaceutical Co.)}

Similar to neratinib, pyrotinib is an orally available irreversible pan-HER targeting TKI. In phase 1, monotherapy with pyrotinib showed an ORR of 50\% (83.3\% in trastuzumabnaive and $33.3 \%$ in trastuzumab-pretreated patients) and a CBR of $61 \%$ in HER2-positive MBC. Common pyrotinibrelated adverse events included diarrhea (44.7\%), nausea (13.2\%), stomatitis $(13.2 \%)$ and fatigue $(10.5 \%)$. The only grade 3 adverse event was diarrhea (13.2\%), which also defined the dose-limiting toxicity. ${ }^{41}$

In a randomized phase 2 trial, Chinese patients with HER2-positive MBC previously treated with taxanes, anthracyclines and/or trastuzumab were randomly assigned to pyrotinib plus capecitabine $(n=65)$ or lapatinib plus capecitabine $(\mathrm{n}=62)$. The ORR was $78.5 \%$ with pyrotinib and $57.1 \%$ with lapatinib $(\mathrm{P}=0.01)$. The median PFS was 18.1 vs 7.0 months (adjusted HR 0.36; 95\% CI $0.23-0.58 ; \mathrm{P}<0.001)^{42}$

These data were confirmed in the phase 3 trial PHOEBE, recently presented at the virtual ASCO annual meeting 2020. ${ }^{43}$ In this trial, 266 patients with HER2- positive MBC pretreated with trastuzumab, taxanes and/ or anthracyclines received either pyrotinib (400 mg/d QD) plus capecitabine $\left(1000 \mathrm{mg} / \mathrm{m}^{2} \mathrm{~d} 1-14\right)$ or lapatinib plus capecitabine at standard doses. Most of the patients (79\%) suffered from visceral metastases, $39 \%$ and $45 \%$ of patients were treated in first- and second-line, respectively. Median PFS assessed by the blinded independent central review board was significantly longer in the pyrotinib arm compared to the lapatinib arm: 12.5 vs 6.8 months (HR 0.39 ; 95\% CI 0.27-0.56; one-sided $\mathrm{P}<0.0001$ ). All subgroups derived comparable benefit from the new compound. ORR and CBR were $67.2 \%$ and $73.1 \%$ in the pyrotinib plus capecitabine arm and $51.5 \%$ and $59.1 \%$ in the control arm, respectively. OS data were not mature, however, there was a strong trend towards an improved OS in the pyrotinib arm (HR 0.46; 95\% CI 0.22-0.99).

As expected, due to the overlapping toxicities of the two compounds, the main side effect was diarrhea. Almost all patients $(94.8 \%)$ experienced any grade of diarrhea in the experimental arm vs $62.1 \%$ in the control arm; grade 3 diarrhea was recorded in $30.6 \%$ and $8.3 \%$, respectively. At least, diarrhea was reported to be of short duration, reversible and rarely led to treatment discontinuation. No primary prophylaxis for diarrhea was prescribed in this trial. Other grade 3 side effects were hand-foot syndrome (16.4\% vs $15.2 \%)$ and vomiting (6\% vs $0.8 \%){ }^{43}$

Several phase 2 and 3 trials in different disease stages are currently ongoing in China (NCT04033172, NCT0 3933982, NCT03910712, NCT03876587, NCT04254263, NCT04255056, NCT04126525, NCT03735966, NCT044 07988, NCT03863223, NCT03588091, NCT04290793 and several more).

\section{Tucatinib (Seattle Genetics)}

Tucatinib is a third generation, orally available, highly selective inhibitor of the HER2 tyrosine kinase. Encouraging data for tucatinib had already been observed in a phase I dose-escalation trial, which determined the recommended phase 2 dose and evaluated the safety and antitumor activity of tucatinib in combination with trastuzumab and capecitabine in patients with HER2-positive MBC with or without brain metastases. ${ }^{44}$

In the HER2CLIMB phase II trial, patients with HER2positive $\mathrm{MBC}$ were randomly assigned in a 2:1 ratio to receive either tucatinib or placebo in combination with trastuzumab and capecitabine. ${ }^{45}$ All patients had received prior treatment with trastuzumab, pertuzumab and T-DM1. Patients with brain metastases were included and well 
balanced between the two study arms. The estimated PFS at one year was $33.1 \%$ in the tucatinib-combination arm and $12.3 \%$ in the placebo-combination arm. The median PFS was 7.8 and 5.6 months, respectively. The HR for disease progression or death was 0.54 (95\% CI $0.42-0.71$; $\mathrm{P}<0.001$ ), corresponding to a $46 \%$ risk reduction in favor of tucatinib. Similar results were observed in terms of two year-estimated OS ( $44.9 \%$ vs $26.6 \%$ ), with a median OS of 21.9 months in the tucatinib group and 17.4 in the placebo group (HR 0.66; 95\% CI 0.50-0.88; $\mathrm{P}=0.005$ ). In the subgroup analysis for both PFS and OS, all predefined subgroups derived benefit from the new TKI.

As a matter of particular interest, patients with brain metastases also benefited from the addition of tucatinib. Among this subgroup, indeed, the risk of disease progression or death was reduced by $52 \%$ in patients receiving tucatinib compared to those receiving placebo (HR 0.48 95\% CI 0.34-0.69; $\mathrm{P}<0.001$ ).

Both therapy arms experienced toxicity, diarrhea being the most frequent one. Due to the low affinity of tucatinib to other receptors of the HER family besides HER2, the rate of high-grade diarrhea was limited in the tucatinib arm (12.9 vs $8.6 \%)$. The majority of patients in the tucatinib arm (80.9\%), however, experienced grade 1 diarrhea. The most common additional AEs in the tucatinib arm were hand-food-syndrome, nausea, fatigue and vomiting - the majority of them being low grade (1-2). Furthermore, a transient elevation of liver enzyme levels (AST, ALT, Bilirubin) was observed. Of note, the discontinuation rate of tucatinib was only $5.7 \% .{ }^{45}$

Based on the results of the HER2CLIMB study, the FDA approved the simultaneous blockade of HER2s internal and external domain with tucatinib and trastuzumab in combination with capecitabine for the treatment of patients with or without brain metastases, who have had received one or more prior anti-HER2-based regimens in the metastatic setting.

\section{CDK4/6 Inhibitors}

The efficacy and tolerance of CDK4/6-inhibitors in combination with endocrine therapy has been widely demonstrated in patients with HR-positive, HER2-negative MBC. In HR-positive, HER2-positive (triple-positive) breast cancer, there is a well-known crosstalk between HER2 and HR signaling pathways leading to reciprocal treatment resistance. Moreover, preclinical studies suggest that the cyclin $\mathrm{D} / \mathrm{CDK} 4 / 6$ pathway may also have a pivotal role in anti-HER2 treatment resistance. This fact led to the belief that CDK4/6 inhibitors may enhance the activity of antiHER2 agents and overcome the resistance to HER2blockade.

\section{Palbociclib (Pfizer)}

The Phase 2 PATRICIA trial (NCT02448420) evaluated the combination of palbociclib and trastuzumab \pm letrozole in patients with HER2-positive MBC, who had received a median of three prior lines of anti-HER2-based regimens. ${ }^{46}$ Palbociclib was administered at $200 \mathrm{mg} /$ day for 14 days of 21-day cycles. In total, 45 patients were enrolled and divided into 3 cohorts: estrogen receptor (ER)-negative (cohort A), ER-positive (cohort B1) and ER-positive treated with letrozole (cohort B2). A PFS of $40 \%$ at 6 months was required to access Part 2 of the study according to the Simon 2-stage design. The six month-PFS rates were $33.3 \%, 40.0 \%, 53.3 \%$ in cohorts $\mathrm{A}, \mathrm{B} 1$ and $\mathrm{B} 2$, respectively. Therapy was generally well tolerated, since the most common grade 3-4 adverse events were neutropenia and thrombocytopenia, as expected. The genomic assay PAM50 was performed in order to find an association between PFS and molecular intrinsic subtypes. In all cohorts, patients with luminal subtypes obtained a longer PFS (12.4 vs 4.1 months; adjusted HR 0.37; $\mathrm{P}=0.052$ ) and a higher $\mathrm{CBR}(73 \%$ vs $31 \%$; $\mathrm{P}=0.031)$ compared with nonluminal tumors.

This interim result supports the combination of CDK4/ 6 inhibitors with trastuzumab, especially in luminal HER2positive disease. Consequently, two additional cohorts will be included: patients with ER-positive, HER2-positive MBC and luminal subtype according to PAM50 will be randomized to receive trastuzumab, palbociclib and endocrine therapy (cohort $\mathrm{C} 1$ ) or trastuzumab plus physician's choice treatment, including T-DM1 or chemotherapy (cohort $\mathrm{C} 2$ ). ${ }^{46}$

\section{Abemaciclib (Eli Lilly and Company)}

Tolaney and colleagues presented at the 2019 European Society of Medical Oncology (ESMO) Meeting the primary results of the MonarcHER trial, a randomized 3-arm, phase 2 study, comparing the CDK4/6 inhibitor abemaciclib in combination with trastuzumab and fulvestrant (arm A) to abemaciclib plus trastuzumab (arm B) and trastuzumab plus physician's choice chemotherapy (arm C). ${ }^{47}$ The trial included postmenopausal patients with HR+/HER2+ MBC, who had received a taxane and at least two prior anti-HER2 targeting therapies, including T-DM1. The median PFS was longer in arm A (8.3 months) with 
a statistically significant absolute difference of 2.6 months when compared to arm C (HR 0.67; 95\% CI 0.45-1.00; $\mathrm{P}=0.051$ [pre-specified 2-sided alpha of 0.2 ]). No difference was observed between arm B and C (median PFS 5.7 vs 5.7 months; HR $0.94 ; 95 \%$ CI $0.64-1.38$; $\mathrm{P}=0.77$ ). Promising results were also observed in terms of ORR with rates of $35.4 \%, 16.5 \%$ and $22.8 \%$ in arms $\mathrm{A}, \mathrm{B}$ and $\mathrm{C}$, respectively. The safety analysis confirmed the known safety profile of abemaciclib: neutropenia was the most common grade 3-4 treatment-related AE among all groups $(27 \%, 22 \%$ and $26 \%$ of patients in groups A, B and C, respectively), along with leukopenia, thrombocytopenia and diarrhea. Two treatment associated deaths occurred, one due to pulmonary fibrosis in group B and one due to febrile neutropenia in group $\mathrm{C}^{47}$

As a consequence of the primary results of this study, abemaciclib in combination with trastuzumab and fulvestrant could be an appealing chemotherapy-free treatment option in patients with heavily pretreated HR+/HER2+ MBC. However, due to the missing fourth arm consisting of fulvestrant plus trastuzumab, the contribution of abemaciclib to this PFS-benefit cannot clearly be estimated, since the major effect was seen in the fulvestrant containing arm, but not in the abemaciclib plus trastuzumab arm.

\section{Immunotherapy}

Another approach to improve outcomes in HER2-positive $\mathrm{MBC}$ is the implementation of immunotherapy. Checkpoint inhibitors (CPI) targeting the programmed cell death protein 1 (PD-1) or its ligand PD-L1 have been approved as monotherapy for several solid tumors like melanoma, kidney cancer and a subgroup of non-small cell lung cancer (NCSCL). In breast cancer, however, CPI-monotherapy has only shown limited efficacy, even in triple-negative breast cancer (TNBC), the most immunogenic subtype. ${ }^{48-}$ ${ }^{50}$ In contrast, in combination with chemotherapy, specifically nab-paclitaxel, the PD-L1 inhibitor atezolizumab improved PFS and OS in patients with PD-L1-positive TNBC and has now been approved for first-line treatment of metastatic TNBC. ${ }^{51}$ In HER2-positive MBC, to date two phase 2 trials have been presented combining CPI with either trastuzumab or T-DM1.

\section{Pembrolizumab (Anti-PD-I mAb; Merck Sharp \& Dohme Corp.)}

In the single-arm, multicenter, phase 1b-2 PANACEA trial, patients with HER2-positive MBC and documented progression during previous trastuzumab-based therapy, received the anti-PD-1 mAb pembrolizumab plus trastuzumab. ${ }^{52}$ The primary endpoint of the phase 2 part of the study was the proportion of PD-L1-positive patients achieving an objective response, which was achieved in 6 of 40 patients $(15 \%)$. No objective responses were seen among PD-L1-negative patients. Disease control was achieved in 10 patients $(25 \%)$ in this cohort. In an exploratory analysis, there were significantly more tumorinfiltrating lymphocytes (TILs) in patients with response or stable disease compared to patients with progressive disease as best response. Median PFS was 2.7 months (90\% CI 2.6-4.0) with an estimated 6-month PFS rate of $25 \%$ and a 12 -month PFS rate of $12 \%$. Median OS has not been reached (90\% CI 13.1-not reached).

Immune-mediated AEs were recorded in 11 (19\%) of 58 patients including hypothyroidism $(\mathrm{n}=2)$, hyperthyroidism $(n=2)$, pneumonitis $(n=2)$ and autoimmune hepatitis $(n=2)$. One patient died from treatment-related LambertEaton syndrome. $^{52}$

\section{Atezolizumab (Anti-PD-LI mAb; Roche)}

In the KATE2 phase 2 trial, patients with HER2-positive advanced breast cancer progressing on palliative therapy with a taxane plus trastuzumab or within 6 months of adjuvant therapy, were randomly assigned to either T-DM1 plus placebo or T-DM1 plus the anti-PD-L1 mAb atezolizumab (1200 mg flat-dose every 3 weeks IV). ${ }^{53}$ Randomization was stratified by PD-L1 status (less vs equal or more than $1 \%$ PD-L1 expression on immune cells IC), world region (Western Europe vs North America vs rest of the world) and presence or absence of liver metastases.

Disappointingly, the primary endpoint was not reached: the median investigator-assessed PFS in the ITT population was 8.2 months in the T-DM1 plus atezolizumab group and 6.8 months in the T-DM1 plus placebo group without reaching statistical significance (stratified HR $0.82 ; 95 \%$ CI $0.55-1.23 ; \mathrm{P}=0.33) .{ }^{53}$ Similarly, there was only a numerically OS advantage, one of the secondary endpoints (HR 0.74, 95\% CI 0.42-1.30; 1-year OS-rate: $89.1 \%$ vs $89.0 \%$ ). An exploratory and post-hoc analysis suggested a greater benefit for the PD-L1-positive subgroup (IC $\geq 1 \%$ ) for PFS (HR 0.60; 95\% CI 0.32-1.11) and OS (HR 0.55; 95\% CI $0.22-1.38$ ), respectively. ${ }^{54}$ No new safety signals were reported for atezolizumab in this trial, however, high-grade thrombocytopenia was more 
frequent in the atezolizumab arm compared to the placebo arm (grade 3-4: $14 \%$ vs $6 \%$ ).

Atezolizumab is currently being tested in a randomized phase III trial against placebo in combination with paclitaxel, trastuzumab and pertuzumab in first-line (NRGBR004 trial; NCT03199885). The AVIATOR phase 2 study investigates trastuzumab and vinorelbine with avelumab or avelumab plus utomilumab, a novel 41BB/ CD137 agonist, in patients with HER2-positive MBC who progressed on prior trastuzumab and pertuzumab (NCT03414658).

Other immunotherapeutic strategies, as chimeric antigen receptor $\mathrm{T}$ (CAR-T) cells, interleukin-2 fusion proteins or vaccination against HER2 peptides are currently under investigation. Since to date, there are no or only limited clinical data available, their chances for success in the drug development process are hard to predict yet.

\section{Conclusion}

Major progress has been made in the treatment of HER2positive $\mathrm{MBC}$ in recent years. A median OS of more than 4.5 years, as seen in the CLEOPATRA phase 3 trial with docetaxel plus trastuzumab and pertuzumab, was unthinkable before the introduction of anti-HER $2 \mathrm{mAb}$. After failure of these two drugs, the new TKI tucatinib in combination with capecitabine has prolonged PFS and OS and has achieved objective response in $40 \%$ of patients in the randomized HER2CLIMB phase II trial. Furthermore, this small molecule led to intracranial responses and a survival advantage in patients with brain metastases, the major site of concern in HER2-positive breast cancer. In addition, new ADCs like trastuzumab deruxtecan and trastuzumab duocarmazine have shown impressing response rates with deep and long-lasting remissions in already heavily pretreated patients. The latter findings raise hope for further improvement of survival in this breast cancer subtype. In addition, adding checkpoint inhibitors or bispecific antibodies to the treatment armamentarium of HER2-positive $\mathrm{MBC}$ might enable persistent responses and even cure in this disease.

Such rapid developments frequently complicate therapy sequencing and hinder the establishment of clear treatment guidelines. However, these difficulties are much easier to overcome than those raised by the limited treatment options as in triple-negative breast cancer, which is still far away from the success story of HER2-positive breast cancer treatment.

\section{Abbreviations}

ADC, antibody-drug conjugate; ADCC, antibodydependent cell-mediated cytotoxicity; AEs, adverse events; ASCO, American Society of Clinical Oncology; CAR-T, chimeric antigen receptor T cells; CBR, clinical benefit rate; CDK4/6, cyclin-dependent kinase 4 and 6; CI, confidence interval; CPI, checkpoint inhibitor; $\mathrm{CR}$, complete remission; DCR, disease control rate; EGFR, epidermal growth factor receptor; EMA, European Medicines Agency; ER, estrogen receptor; ESMO, European Society of Medical Oncology; Fc $\gamma R$, Fc-gamma receptor; FDA, Food and Drug Administration; G, grade; HER2, human epidermal growth factor receptor 2; HER3, human epidermal growth factor receptor 3; HER4, human epidermal growth factor receptor 4; HR, hazard ratio; HR, hormone receptor; IC, immune cells; ILD, interstitial lung disease; IRR, infusion-related reactions; ITT, intention-to-treat; IV, intravenous; $\mathrm{mAb}$, monoclonal antibody; $\mathrm{MBC}$, metastatic breast cancer; MMAE, monomethyl auristatin E; NRG-1, neuregulin-1; NSABP, National Surgical Adjuvant Breast and Bowel Project; NSCLC, non-small cell lung cancer; ORR, overall response rate; OS, overall survival; PD-L1, programmed death receptor ligand 1; PFS, progression-free survival; PR, partial remission; SABCS, San Antonio Breast Cancer Symposium; SAEs, serious adverse events; SD, stable disease; TDB, T cell-dependent bispecific; TILs, tumor infiltrating lymphocytes; TKI, tyrosine kinase inhibitor; TNBC, triple-negative breast cancer.

\section{Author Contributions}

Conception and design: Simon P. Gampenrieder, Richard Greil.

Collection and assembly of data: Simon P. Gampenrieder, Gabriel Rinnerthaler, Vanessa Castagnaviz.

Data analysis and interpretation: All authors.

Manuscript writing: Simon P. Gampenrieder, Vanessa Castagnaviz.

Critical revising of the manuscript: All authors.

Final approval of manuscript: All authors.

All authors took part in revising or critically reviewing the article; gave final approval of the version to be published; have agreed on the journal to which the article has been submitted and agree to be accountable for all aspects of the work.

\section{Disclosure}

Gabriel Rinnerthaler declares the following relations: Consulting or Advisory Role: Pierre Fabre, Roche, 
Novartis, Pfizer, Eli Lilly; Speakers Bureau: Amgen, AstraZeneca, Novartis, Bristol-Myers Squibb, Roche, Pfizer, Eli Lilly; Research Funding: Roche; Travel, Accommodation, Expenses: Roche, Novartis, Amgen, Pfizer, Bristol-Myers Squibb;

Simon Peter Gampenrieder declares the following relations: Honoraria: Novartis, Roche, BMS, AstraZeneca, MSD; Consulting or Advisory Role: Roche, Novartis, Pfizer, Lilly, AstraZeneca, MSD; Research Funding: Roche; Travel, Accommodation, Expenses: Roche, Amgen, Shire, Novartis, Pfizer, Bayer, Celgene, Daiichi Sankyo.

Vanessa Castagnaviz has no conflicts of interest to declare.

Richard Greil declares the following relations: Honoraria: Celgene, Roche, Merck, Takeda, AstraZeneca, Novartis, Amgen, BMS, MSD, Sandoz, Abbvie, Gilead, Daiichi Sankyo; Consulting or Advisory Role: Celgene, Novartis, Roche, BMS, Takeda, Abbvie, AstraZeneca, Janssen, MSD, Merck, Gilead, Daiichi Sankyo;; Research Funding: Celgene, Merck, Takeda, AstraZeneca, Novartis, Amgen, BMS, MSD, Sandoz, Gilead, Roche; Travel, Accommodation, Expenses: Roche, Amgen, Janssen, AstraZeneca, Novartis, MSD, Celgene, Gilead, BMS, Abbvie, Daiichi Sankyo.

\section{References}

1. Sørlie T, Perou CM, Tibshirani R, et al. Gene expression patterns of breast carcinomas distinguish tumor subclasses with clinical implications. Proc Natl Acad Sci U S A. 2001;98(19):10869-10874. doi:10.1073/pnas.191367098

2. Curigliano G, Burstein HJ, P Winer E, et al. De-escalating and escalating treatments for early-stage breast cancer: the St. Gallen International Expert Consensus Conference on the Primary Therapy of Early Breast Cancer 2017. Ann Oncol. 2019;30(7):1181. doi:10.1093/annonc/mdy537

3. Howlader N, Altekruse SF, Li CI, et al. US incidence of breast cancer subtypes defined by joint hormone receptor and HER2 status. J Natl Cancer Inst. 2014;106(5). doi:10.1093/jnci/dju055.

4. Sundquist M, Brudin L, Tejler G. Improved survival in metastatic breast cancer 1985-2016. Breast (Edinburgh, Scotland). 2017;31: 46-50. doi:10.1016/j.breast.2016.10.005

5. Slamon DJ, Leyland-Jones B, Shak S, et al. Use of chemotherapy plus a monoclonal antibody against HER2 for metastatic breast cancer that overexpresses HER2. $N$ Engl J Med. 2001;344(11):783-792. doi:10.1056/NEJM200103153441101

6. Andersson M, Lidbrink E, Bjerre K, et al. Phase III randomized study comparing docetaxel plus trastuzumab with vinorelbine plus trastuzumab as first-line therapy of metastatic or locally advanced human epidermal growth factor receptor 2-positive breast cancer: the HERNATA study. J Clin Oncol. 2011;29(3):264-271. doi:10.1200/ JCO.2010.30.8213

7. Valero V, Forbes J, Pegram MD, et al. Multicenter phase III randomized trial comparing docetaxel and trastuzumab with docetaxel, carboplatin, and trastuzumab as first-line chemotherapy for patients with HER2-gene-amplified metastatic breast cancer (BCIRG 007 study): two highly active therapeutic regimens. J Clin Oncol. 2011;29(2):149-156.
8. Swain SM, Baselga J, Kim SB, et al. Pertuzumab, trastuzumab, and docetaxel in HER2-positive metastatic breast cancer. $N$ Engl J Med. 2015;372(8):724-734. doi:10.1056/NEJMoa1413513

9. Lin NU, Dieras V, Paul D, et al. Multicenter phase II study of lapatinib in patients with brain metastases from HER2-positive breast cancer. Clin Cancer Res. 2009;15(4):1452-1459. doi:10.1158/10780432.CCR-08-1080

10. Scheuer W, Friess T, Burtscher H, Bossenmaier B, Endl J, Hasmann M. Strongly enhanced antitumor activity of trastuzumab and pertuzumab combination treatment on HER2-positive human xenograft tumor models. Cancer Res. 2009;69(24):9330-9336. doi:10.1158/0008-5472.CAN-08-4597

11. Beck A, Goetsch L, Dumontet C, Corvaia N. Strategies and challenges for the next generation of antibody-drug conjugates. Nat Rev Drug Discov. 2017;16(5):315-337. doi:10.1038/nrd.2016.268

12. Verma S, Miles D, Gianni L, et al. Trastuzumab emtansine for HER2-positive advanced breast cancer. $N$ Engl J Med. 2012;367 (19):1783-1791. doi:10.1056/NEJMoa1209124

13. Krop IE, Kim SB, Gonzalez-Martin A, et al. Trastuzumab emtansine versus treatment of physician's choice for pretreated HER2-positive advanced breast cancer (TH3RESA): a randomised, open-label, phase 3 trial. Lancet Oncol. 2014;15(7):689-699. doi:10.1016/S14702045(14)70178-0

14. Rugo HS, Im S-A, Cardoso F, et al. Phase 3 SOPHIA study of margetuximab + chemotherapy vs trastuzumab + chemotherapy in patients with HER2+ metastatic breast cancer after prior anti-HER2 therapies: second interim overall survival analysis. Presented at: San Antonio Breast Cancer Symposium SABCS 2019, abstract GS1-02.

15. Hamilton EP, Petit T, Pistilli B, et al. Clinical activity of MCLA-128 (zenocutuzumab), trastuzumab, and vinorelbine in HER2 amplified metastatic breast cancer (MBC) patients (pts) who had progressed on anti-HER2 ADCs. Presented at the ASCO Annual Meeting 2020, abstract 3093.

16. Modi S, Saura C, Yamashita T, et al. Trastuzumab Deruxtecan in previously treated HER2-positive breast cancer. $N$ Engl J Med. 2020;382(7):610-621. doi:10.1056/NEJMoa1914510

17. Burstein HJ, Sun Y, Dirix LY, et al. Neratinib, an irreversible ErbB receptor tyrosine kinase inhibitor, in patients with advanced ErbB2-positive breast cancer. J Clin Oncol. 2010;28(8):1301-1307. doi:10.1200/JCO.2009.25.8707

18. Martin M, Bonneterre J, Geyer CE Jr, et al. A phase two randomised trial of neratinib monotherapy versus lapatinib plus capecitabine combination therapy in patients with HER2+ advanced breast cancer. Eur J Cancer. 2013;49(18):3763-3772. doi:10.1016/j. ejca.2013.07.142

19. Awada A, Colomer R, Inoue K, et al. Neratinib plus paclitaxel vs trastuzumab plus paclitaxel in previously untreated metastatic ERBB2-positive breast cancer: the NEfERT-T randomized clinical trial. JAMA Oncol. 2016;2(12):1557-1564. doi:10.1001/ jamaoncol.2016.0237

20. Abraham J, Montero AJ, Jankowitz RC, et al. Safety and Efficacy of T-DM1 plus neratinib in patients with metastatic HER2-positive breast cancer: NSABP foundation trial FB-10. J Clin Oncol. 2019;37(29):2601-2609. doi:10.1200/JCO.19.00858

21. Freedman RA, Gelman RS, Anders CK, et al. TBCRC 022: a Phase II trial of neratinib and capecitabine for patients with human epidermal growth factor receptor 2-positive breast cancer and brain metastases. J Clin Oncol. 2019;37(13):1081-1089. doi:10.1200/JCO.18.01511

22. Saura C, Oliveira M, Feng Y-H, et al. Neratinib + capecitabine versus lapatinib + capecitabine in patients with HER2+ metastatic breast cancer previously treated with $\geq 2$ HER2-directed regimens: findings from the multinational, randomized, phase III NALA trial. J Clin Oncol. 2019;37(15_suppl):1002. 
23. Ma F, Ouyang Q, Li W, et al. Pyrotinib or Lapatinib Combined With Capecitabine in HER2-positive metastatic breast cancer with prior taxanes, anthracyclines, and/or trastuzumab: a randomized, phase II study. J Clin Oncol. 2019;37(29):2610-2619. doi:10.1200/JCO. 19.00108

24. Xu B, Yan M, Ma F, et al. Pyrotinib or lapatinib plus capecitabine for HER 2 + metastatic breast cancer (PHOEBE): a randomized phase III trial. Presented at the ASCO Annual Meeting 2012 Abstract 1003.

25. Murthy RK, Loi S, Okines A, et al. Tucatinib, trastuzumab, and capecitabine for HER2-positive metastatic breast cancer. $N$ Engl $J$ Med. 2020;382(7):597-609. doi:10.1056/NEJMoa1914609

26. Tolaney SM, Wardley AM, Zambelli S, et al. Abemaciclib plus trastuzumab with or without fulvestrant versus trastuzumab plus standard-of-care chemotherapy in women with hormone receptor-positive, HER2-positive advanced breast cancer (monarcHER): a randomised, open-label, phase 2 trial. Lancet Oncol. 2020;21(6):763-775. doi:10.1016/S1470-2045(20)30112-1

27. Ciruelos E, Villagrasa P, Paré L, et al. SOLTI-1303 PATRICIA phase II trial (STAGE 1) - palbociclib and trastuzumab in postmenopausal patients with HER2-positive metastatic breast cancer. Presented at the San Antonio Breast Cancer Symposium SABCS 2018, abstract PD303 .

28. Loi S, Giobbie-Hurder A, Gombos A, et al. Pembrolizumab plus trastuzumab in trastuzumab-resistant, advanced, HER2-positive breast cancer (PANACEA): a single-arm, multicentre, phase 1b-2 trial. Lancet Oncol. 2019;20(3):371-382. doi:10.1016/S14702045(18)30812-X

29. Emens LA, Esteva F, Beresford M, et al. Results from KATE2, a randomized phase 2 study of atezolizumab (atezo)+trastuzumab emtansine (T-DM1) vs placebo (pbo)+T-DM1 in previously treated HER2+ advanced breast cancer (BC). Presented at the San Antonio Breast Cancer Symposium SABCS 2019, abstract PD3-01; 2019:79.

30. Emens LA, Esteva FJ, Beresford M, et al. Overall survival (OS) in KATE2, a phase II study of programmed death ligand 1 (PD-L1) inhibitor atezolizumab (atezo)+trastuzumab emtansine (T-DM1) vs placebo (pbo)+T-DM1 in previously treated HER2+ advanced breast cancer (BC). Presented at the European Society of Medical Oncology (ESMO) Congress 2019, abstract 305O; 2019.

31. Nordstrom JL, Gorlatov S, Zhang W, et al. Anti-tumor activity and toxicokinetics analysis of MGAH22, an anti-HER2 monoclonal antibody with enhanced Fcgamma receptor binding properties. Breast Cancer Res. 2011;13(6):R123. doi:10.1186/bcr3069

32. Hamblett KJ, Barnscher SD, Davies RH, et al. Abstract P6-17-13: ZW49, a HER2 targeted biparatopic antibody drug conjugate for the treatment of HER2 expressing cancers. Presented at the san Antonio Breast Cancer Symposium SABCS 2018, abstract P6-17-13.

33. Meric-Bernstam F, Beeram M, Mayordomo JI, et al. Single agent activity of ZW25, a HER2-targeted bispecific antibody, in heavily pretreated HER2-expressing cancers. Presented at the ASCO Annual Meeting 2018 Abstract 2500.

34. Alsina M, Boni V, Schellens JHM, et al. First-in-human phase 1/2 study of MCLA-128, a full length IgG1 bispecific antibody targeting HER2 and HER3: final phase 1 data and preliminary activity in HER2+ metastatic breast cancer (MBC). Presented at the ASCO Annual Meeting 2017 Abstract 2522.

35. Pistilli B, Wildiers H, Hamilton EP, et al. Clinical activity of MCLA-128 (zenocutuzumab) in combination with endocrine therapy (ET) in ER+/HER2-low, non-amplified metastatic breast cancer (MBC) patients (pts) with ET-resistant disease who had progressed on a CDK4/6 inhibitor (CDK4/6i). Presented at the ASCO Annual Meeting 2020, abstract 1037.

36. Schram AM, O'Reilly EM, Somwar R, Benayed R, Shameem S Clinical proof-of-concept for MCLA-128, a bispecific HER2/3 antibody therapy, in NRG1 fusion-positive cancers. Presented at the AACR-NCI-EORTC Congress 2019, abstract PR02.
37. Rius Ruiz I, Vicario R, Morancho B, et al. p95HER2-T cell bispecific antibody for breast cancer treatment. Sci Transl Med. 2018;10:461. doi:10.1126/scitranslmed.aat1445

38. Nakada T, Sugihara K, Jikoh T, Abe Y, Agatsuma T. The latest research and development into the antibody-drug conjugate, [fam-] trastuzumab deruxtecan (DS-8201a), for HER2 cancer therapy. Chem Pharm Bull (Tokyo). 2019;67(3):173-185. doi:10.1248/cpb.c1800744

39. Doi T, Shitara K, Naito Y, et al. Safety, pharmacokinetics, and antitumour activity of trastuzumab deruxtecan (DS-8201), a HER2-targeting antibody-drug conjugate, in patients with advanced breast and gastric or gastro-oesophageal tumours: a phase 1 dose-escalation study. Lancet Oncol. 2017;18(11):1512-1522. doi:10.1016/S1470-2045(17)30604-6

40. Banerji U, van Herpen CML, Saura C, et al. Trastuzumab duocarmazine in locally advanced and metastatic solid tumours and HER2-expressing breast cancer: a phase 1 dose-escalation and dose-expansion study. Lancet Oncol. 2019;20(8):1124-1135. doi:10.1016/S1470-2045(19)30328-6

41. Tang W, Deng X, Ou Z, et al. BAT8001, a potent anti-HER2 antibody-drug conjugate with a novel stable linker for the treatment of HER2-positive breast cancer. Presented at the San Antonio Breast Cancer Symposium SABCS 2018, abstract P6-17-39.

42. Wang S, Xu F, Hong R, et al. BAT8001, a potent anti-HER2 antibody drug conjugate with a novel uncleavable linker to reduce toxicity for patients with HER2-positive tumor. Presented at the AACR Annual Meeting 2019, abstract CT053; 2019.

43. Yao X, Jiang J, Wang X, et al. A novel humanized anti-HER2 antibody conjugated with MMAE exerts potent anti-tumor activity. Breast Cancer Res Treat. 2015;153(1):123-133. doi:10.1007/ s10549-015-3503-3

44. Xu B, Wang J, Fang J, et al. Early clinical development of RC48-ADC in patients with HER2 positive metastatic breast cancer. Presented at the San Antonio Breast Cancer Symposium SABCS 2019, abstract PD4-06.

45. Sheng X, Zhou A, Yao X, Shi Y, Luo H A phase II study of RC48-ADC in HER2-positive patients with locally advanced or metastatic urothelial carcinoma. Presented at the ASCO Annual Meeting 2019 Abstract 4509.37.

46. Humphreys RC, Kirtely J, Hewit A, et al. Site specific conjugation of ARX-788, an antibody drug conjugate (ADC) targeting HER2, generates a potent and stable targeted therapeutic for multiple cancers. Presented at the AACR Annual Meeting 2015, abstract 639.

47. Park YH, Ahn HK, Kim J-Y, et al. First-in-human phase I study of ALT-P7, a HER2-targeting antibody-drug conjugate in patients with HER2-positive advanced breast cancer. Presented at the ASCO Annual Meeting 2020 Abstract 3551.

48. Martin M, Holmes FA, Ejlertsen B, et al. Neratinib after trastuzumab-based adjuvant therapy in HER2-positive breast cancer (ExteNET): 5-year analysis of a randomised, double-blind, placebo-controlled, phase 3 trial. Lancet Oncol. 2017;18 (12):1688-1700.

49. Ma F, Li Q, Chen S, et al. Phase I study and biomarker analysis of pyrotinib, a novel irreversible pan-ErbB receptor tyrosine kinase inhibitor, in patients with human epidermal growth factor receptor 2-positive metastatic breast cancer. $J$ Clin Oncol. 2017;35 (27):3105-3112. doi:10.1200/JCO.2016.69.6179

50. Murthy R, Borges VF, Conlin A, et al. Tucatinib with capecitabine and trastuzumab in advanced HER2-positive metastatic breast cancer with and without brain metastases: a non-randomised, open-label, phase 1b study. Lancet Oncol. 2018;19(7):880-888. doi:10.1016/ S1470-2045(18)30256-0

51. Adams S, Schmid P, Rugo HS, et al. Pembrolizumab monotherapy for previously treated metastatic triple-negative breast cancer: cohort A of the phase II KEYNOTE-086 study. Ann Oncol. 2019;30 (3):397-404. doi:10.1093/annonc/mdy517 
52. Nanda R, Chow LQ, Dees EC, et al. Pembrolizumab in patients with advanced triple-negative breast cancer: phase Ib KEYNOTE-012 Study. J Clin Oncol. 2016;34(21):2460-2467. doi:10.1200/JCO.2015.64.8931

53. Cortés J, Lipatov O, Im S-A, Gonçalves A KEYNOTE-119: phase III study of pembrolizumab (pembro) versus single-agent chemotherapy (chemo) for metastatic triple negative breast cancer (mTNBC). Presented at: European Society of Medical Oncology (ESMO) Congress 2019, abstract LBA21.
54. Schmid P, Adams S, Rugo HS, et al. Atezolizumab and Nab-paclitaxel in advanced triple-negative breast cancer. $N$ Engl J Med. 2018;379(22):2108-2121. doi:10.1056/NEJMoa1809615

\section{Publish your work in this journal}

Cancer Management and Research is an international, peer-reviewed open access journal focusing on cancer research and the optimal use of preventative and integrated treatment interventions to achieve improved outcomes, enhanced survival and quality of life for the cancer patient.
The manuscript management system is completely online and includes a very quick and fair peer-review system, which is all easy to use. Visit http://www.dovepress.com/testimonials.php to read real quotes from published authors. 